\title{
A Rare Case of Facial Palsy Due to Mucormycosis
}

\author{
Vaishali Shah', H. Ganapathy', Ram Gopalakrishnan'2, N. Geetha ${ }^{3}$ \\ ${ }^{1}$ ENT Department, Apollo Main Hospital, Chennai, India \\ ${ }^{2}$ Infectious Diseases Department, Apollo Main Hospital, Chennai, India \\ ${ }^{3}$ Pathology Department, Apollo Main Hospital, Chennai, India \\ Email: drvaishalient@gmail.com
}

Received 19 April 2015; accepted 9 May 2015; published 18 May 2015

Copyright (C) 2015 by authors and Scientific Research Publishing Inc.

This work is licensed under the Creative Commons Attribution International License (CC BY). http://creativecommons.org/licenses/by/4.0/

(c) (i) Open Access

\begin{abstract}
A very uncommon instance of facial nerve palsy involving isolated temporal bone with associated uncontrolled diabetes mellitus has been noticed. A 53-year-old diabetic male presented himself with facial asymmetry, ear pain, and discharge in the right ear of one-month duration. Clinical examination revealed grade IV [House-Brackmann] right sided facial palsy, and otoscopy of small central perforation. Clinically acute otitis media with facial palsy diagnosis was made. There was minimal response to medical treatment. As per CT scan and audiometry findings, patient was subjected for exploratory mastoidectomy showing pale granulation tissue involving geniculate ganglion of facial nerve. The histopathology was suggestive of mucormycosis, an unusual presentation in middle ear. The patient was treated with injectable Amphotericin B. This case highlights a rare cause of isolated facial palsy and physicians should be aware of such atypical clinical presentation.
\end{abstract}

\section{Keywords}

Facial Palsy, Diabetes Mellitus, Mucormycosis

\section{Introduction}

The purpose of presenting this case report is to highlight the rare manifestation of mucormycosis causing facial nerve palsy. Many cases of mucormycosis [1]-[4] causing facial palsy have been reported in medical journals published in English literature. Very few publications [1] [2] have documented isolated tympanic bone involvement. Mucormycosis is an emerging fungal infection with a high rate of mortality. Mucormycosis is the term used to describe fungal infections caused by fungi in the order Mucorales, and species in the Mucor, genera rhizopus, absidia and cunninghamella are most often implicated [5]. This disease is often characterized by hyphae 
growing in and around vessels. Mucormycosis frequently involves the paranasal sinuses, brain, or lungs. While oral or cerebral mucormycosis is the most common type of the disease, this infection can also manifest in the gastrointestinal tract, skin, and in other organ systems. In rare cases, the temporal bone may be affected by mucormycosis.

\section{Case Report}

A 53-year-old diabetic male presented himself with facial asymmetry, ear pain, tinnitus and discharge in the right ear of one-month duration. At the onset of presentation, he had taken a conservative line of treatment for the same without any improvement. He was on oral antidiabetic treatment for the past 6 years. Clinical examination revealed absence of right nasolabial fold, deviation of angle of mouth to the left, inability to close the right eye, and asymmetry of face at rest (Figure 1). All these features suggested grade IV facial nerve palsy (HouseBrackmann). Otoscopic examination revealed a small central perforation in the anteroinferior quadrant of the right tympanic membrane. Fistula sign was absent; and there was no evidence of meningitis.

Pure tone audiometry revealed sloping mixed hearing loss in the right ear and sensorineural hearing loss in the left. Culture and sensitivity of purulent discharge from the right ear showed insignificant growth of coagulase negative staphylococci. His fasting and postprandial blood sugar levels were $254 \mathrm{mg} / \mathrm{dl}$ and $375 \mathrm{mg} / \mathrm{dl}$ respectively. HbA1C was $11.7 \%$ suggesting uncontrolled diabetes mellitus. Serum creatinine was $0.9 \mathrm{mg}$. High resolution computed tomography of temporal bone revealed isodense opacification in the right mesotymapanum (Figure 2). Ossicles and external auditory canal were normal. A clinical diagnosis of acute otitis media with facial palsy was made.

Since there was no improvement with conservative line of treatment, the patient was subjected to exploratory mastoidectomy. Intra operatively, pale granulation tissue was seen lying in anterior epitympanum as well in the region close to geniculate ganglion. The white granular mass was removed in piecemeal exposing the facial nerve along the course of horizontal portion. The specimen was then sent for histopathological examination.

Histopathology examination revealed necrotizing subacute inflammation and associated osteomyelitis and aseptate hyphae suggesting mucormycosis (Figure 3). As per advice from infectious disease consultant the patient was started on injection Amphotericin B (1 mg/kg/day in dextrose drip over 22 hours) along with aggressive diabetic management with injectable insulin.

The course of Amphotericin B injection was continued for 24 days. Thereafter, the patient was put on a step down treatment with syrup Posaconazole $400 \mathrm{mg}(10 \mathrm{ml})$ twice daily for 2 weeks. Post treatment clinical monitoring for seven months showed no deterioration in the condition of the patient. With treatment, partial improvement in eye closure and asymmetry of face was noticed (Figure 4), but grading of facial nerve palsy was same.

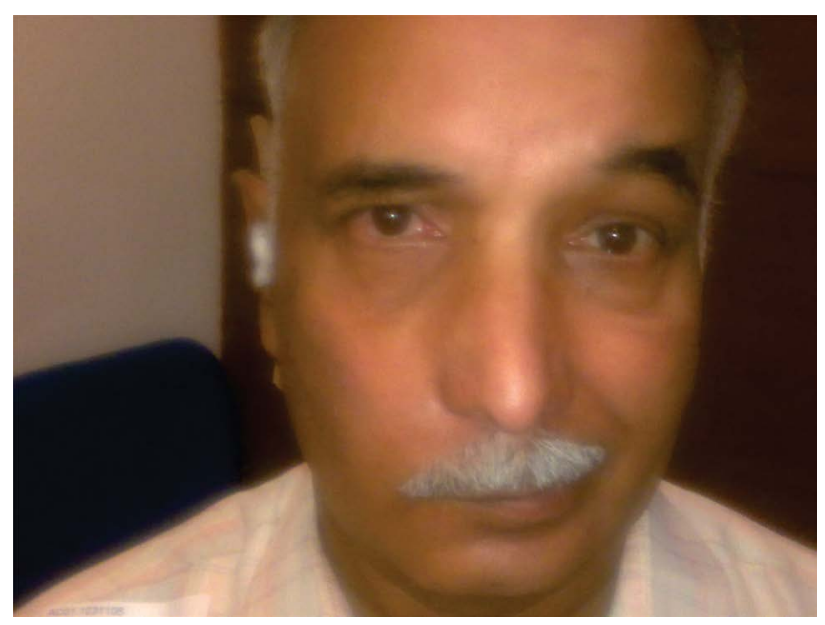

Figure 1. Preoperative clinical picture of patient showing facial asymmetry at rest, absence of right nasolabial fold and deviation of angle of mouth to left side. 


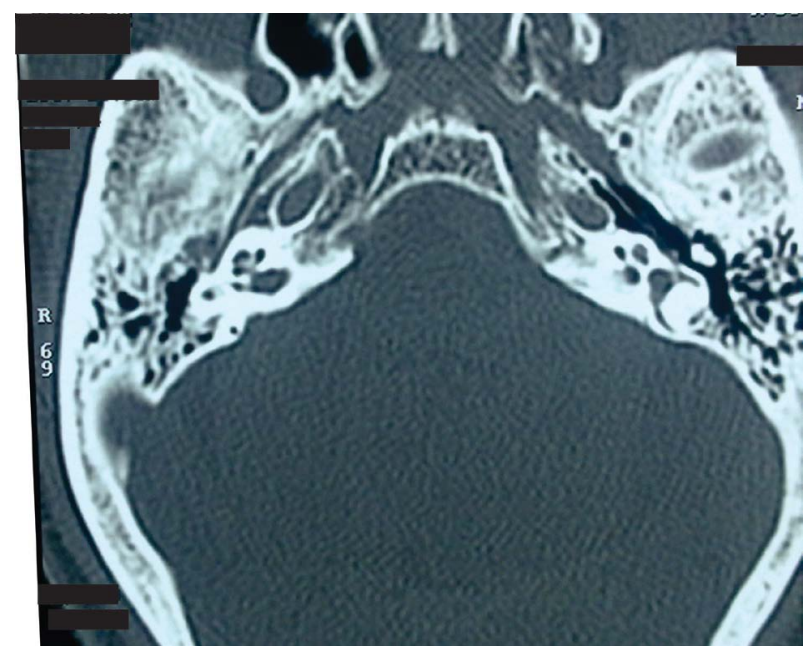

Figure 2. High Resolution CT Temporal bone axial view showing isodenseopacification of right sided mesotympanum, ossicles - intact, no bony erosion.

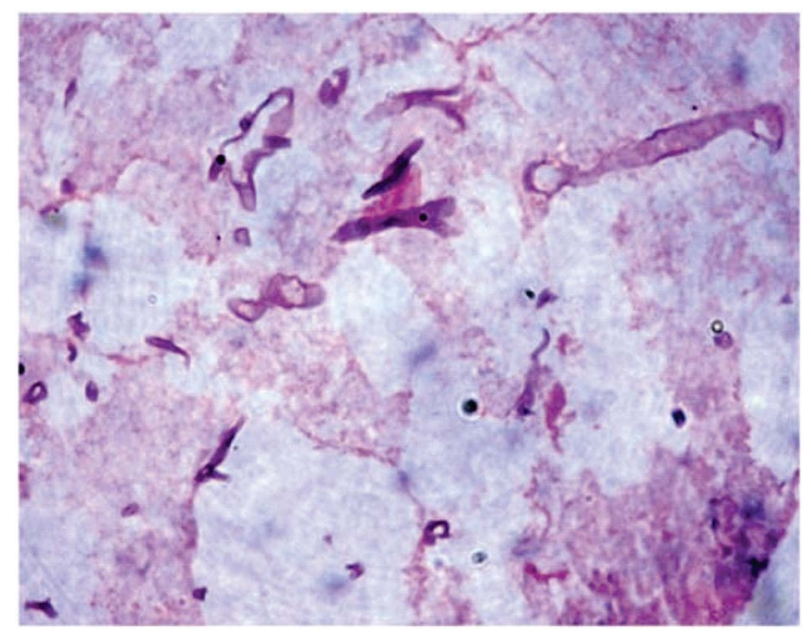

Figure 3. Histopathological slide with Hematoxilin \& Eosin staining with $40 \times$ magnification showing aseptate hyphae.

\section{Discussion}

Mucormycosis is the second most common fungal infection in diabetic patients after aspergillosis [5]. Mucormycosis is caused by subclass of Zygomycetes fungi. Angioinvasive hyphae forms are responsible for tissue invasion and dissemination [5]. Factors associated with an increased risk of mucormycosis include diabetes mellitus, immunosuppression, metabolic acidosis and administration of high dose systemic corticosteroids in solid organs.

In head and neck region, mucormycosis normally manifests itself in the rhino-cerebral area. Very few cases have been reported in the tympanic bone; although rhino-cerebral involvement has been observed quite frequently [3] [4] [6]-[10]. Mucormycosis causing isolated facial nerve paralysis is extremely rare. We reviewed published reports of mucormycosis causing isolated facial paralysis and found only two cases. In one case, horizontal part of facial nerve and external auditory canal were involved [1]. In another case, site of lesion of facial nerve was not mentioned, but external auditory canal was found involved [2]. In our case, the horizontal portion of the facial nerve and the adjacent geniculate ganglion was found involved and external auditory canal was free from disease.

Many articles of mucormycosis spreading from nose or paranasal sinuses to temporal bone were identified 


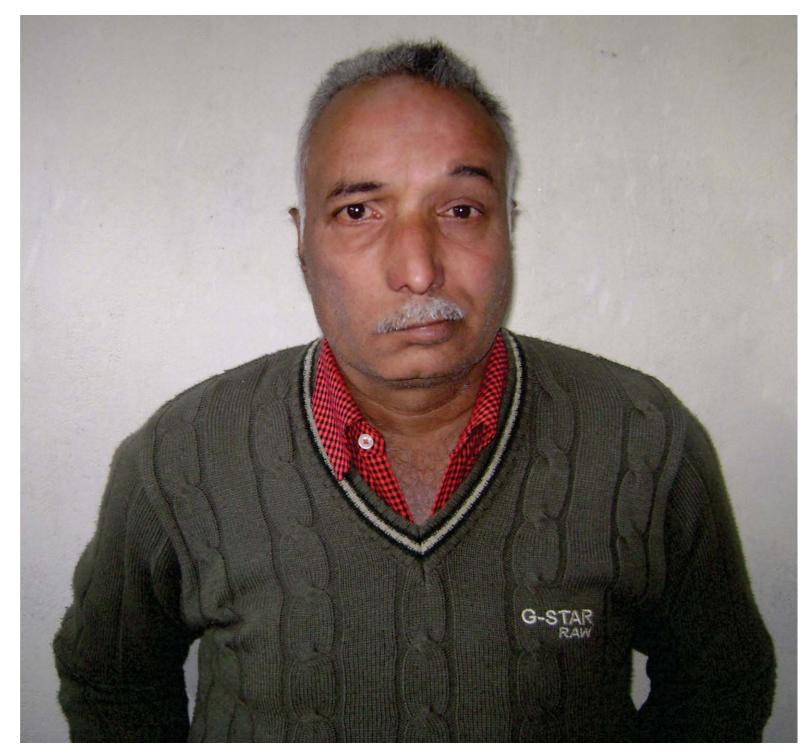

Figure 4. Postoperative clinical picture with slightly improved asymmetry of right-sided face.

while PubMed used as search engine. In two cases, it spread from nasopharynx [6] [7], and in the rest from the paranasal sinuses [3] [4] [8]-[10].

The clinical features of mucormycosis are dependent on the site of involvement. The common causes of facial palsy in diabetic patients include malignant otitis externa, acute on chronic otitis media, malignancy, diabetic neuritis, or Bell's palsy. Fungal infection causing facial palsy is very rare but should be kept in mind before diagnosing it as Bell's palsy. Prognosis of fungal infection is better than malignant otitis externa.

Incidence of Rhino-cerebral mucormycosis with concomitant diabetes mellitus is $60 \%-81 \%$ [11]. Diabetic patients are less likely to improve completely. Recurrence of facial palsy is more common in diabetic patients.

Treatment of mucormycosis is universal. Injectable Amphotericin B is the only drug effective against mucormycosis. Recommended dose of amphotericin is $1-2 \mathrm{mg} / \mathrm{kg} /$ day [5]. Liposomal amphotericin B can be given in high dose of $10 \mathrm{mg} / \mathrm{kg} / \mathrm{day}$. It is to be given for 4 weeks or till there is an improvement. Response rate of Amphotericin B of 50\% has been reported [5]. Posaconazole has been reported with $60 \%-70 \%$ response rate. In our case, as the patient could not afford liposomal Amphotericin B, we gave plain amphotericin B for 4 weeks. Since the patient developed acute renal failure during the treatment, amphotericin was stopped for 12 days and patient was treated with intravenous fluid therapy and potassium supplementation.

Post treatment, the patient continued to show symptoms of grade IV House-Brackmann facial palsy. However, the facial nerve function showed partial improvement in the form of improved eye closure, reduced facial asymmetry at rest although the grading was the same. As per the reported findings in the literature, recovery after grade IV facial nerve paralysis will start from the fourth month onwards [12]. Diabetic patients however tend to show partial recovery only.

\section{Conclusion}

A rare case of facial palsy due to mucormycosis in a diabetic individual has been presented. Physicians should be aware of such atypical clinical presentation. This would facilitate implementation of an early appropriate medical and surgical treatment. Such a treatment regimen would aid disease recovery and better prognosis.

\section{Acknowledgements}

No competing financial interests exist.

\section{Summary}

- Rhino-cerebral involvements are common in mucormycosis involving head and neck regions; 
- This case report highlights rare presentation of mucormycosis causing isolated facial palsy. Physicians should be aware of such atypical clinical presentation;

- Amphotericin B is the universal treatment for the mucormycosis.

\section{References}

[1] Yun, M.W., Lui, C.C. and Chen, W.J. (1994) Facial Paralysis Secondary to Tympanic Mucormycosis: Case Report. American Journal of Otology, 15, 413-414.

[2] Olalla, I., Ortín, M., Hermida, G., Cortés, M.A., Richard, C., Iriondo, A., et al. (1996) Autologous Peripheral Blood Stem Cell Transplantation in a Patient with Previous Invasive Middle Ear Mucormycosis. Bone Marrow Transplant, 18, 1183-1184.

[3] Shekar, V., Sikander, J., Rangdhol, V. and Naidu, M. (2015) Facial Nerve Paralysis: A Case Report of Rare Complication in Uncontrolled Diabetic Patient with Mucormycosis. Journal of Natural Science, Biology and Medicine, 6, 226228. http://dx.doi.org/10.4103/0976-9668.149195

[4] Sachdeva, K. (2013) Rhino-Oculo Cerebral Mucormycosis with Multiple Cranial Nerve Palsy in Diabetic Patient: Review of Six Cases. Indian Journal of Otolaryngology and Head \& Neck Surgery, 65, 375-379. http://dx.doi.org/10.1007/s12070-013-0659-1

[5] Kontoyiannis, D.P. and Lewis, R.E. (2006) Invasive Zygomycosis: Update on Pathogenesis, Clinical Manifestations, and Management. Infectious Diseases Clinics of North America, 20, 581-607.

[6] Oo, M.M., Kutteh, L.A., Koc, O.N., Strauss, M. and Lazarus, H.M. (1998) Mucormycosis of Petrous Bone in an Allogeneic Stem Cell Transplant Recipient. Clinical Infectious Diseases, 27, 1546-1547. http://dx.doi.org/10.1086/517749

[7] Gussen, R. and Canalis, R.F. (1982) Mucormycosis of the Temporal Bone. Annals of Otology, Rhinology \& Laryngology, 91, 27-32. http://dx.doi.org/10.1177/000348948209100108

[8] Nomiya, R., Nomiya, S. and Paparella, M.M. (2008) Mucormycosis of the Temporal Bone. Otology and Neurotology, 29, 1041-1042. http://dx.doi.org/10.1097/MAO.0b013e31817d0200

[9] Hamilton, J.F., Bartkowski, H.B. and Rock, J.P. (2003) Management of CNS Mucormycosis in the Pediatric Patient. Pediatric Neurosurgery, 38, 212-215. http://dx.doi.org/10.1159/000069101

[10] Buhl, M.R., Joseph, T.P., Snelling, B.E. and Buhl, L. (1992) Temporofacial Zygomycosis in a Pregnant Woman. Infection, 20, 230-232. http://dx.doi.org/10.1007/BF02033066

[11] Gupta, S., Koirala, J. and Khardori, R. (2007) Infections in Diabetes Mellitus and Hyperglycemia. Infectious Diseases Clinics of North America, 21, 617-638. http://dx.doi.org/10.1016/j.idc.2007.07.003

[12] Bibas, T., Jiang, D. and Gleeson, M.J. (2008) Disorders of the Facial Nerve. In: Scott-Browne's Otorhinolaryngology, Head and Neck Surgery, 7th Edition, Vol. 3, Chap. 241c, Hodder Arnold, Great Britain, 3870-3894. 